\title{
Biocides Resistance Profiles of Biofilm Forming Bacteria of Dairy Niche and their Control
}

\author{
Mitali Makwana ${ }^{1 *}$, Chand Ram Grover ${ }^{2}$ and Narendra Kumar ${ }^{2}$ \\ ${ }^{1}$ Dairy Microbiology Division, Anand Agricultural University, Anand-3881 10, Gujarat, India \\ ${ }^{2}$ Dairy Microbiology Division, National Dairy Research Institute, \\ Karnal-132001, Haryana, India \\ *Corresponding author
}

\section{A B S T R A C T}

Microbial biofilms are of special concern to dairy and food industry, as biofilms on raw materials or food contact surfaces represent possible sources of contamination with

Keywords

Biofilm, Irreversible,

Efficiency, Resistance,

Persistence

Article Info

Accepted:

10 January 2018

Available Online:

10 February 2018 spoilage or pathogenic microbes. The present study was carried out to evaluate biocide resistance profiles of biofilm forming bacteria of dairy niche towards commonly used biocides and their control in biofilm formation. Genetically confirmed 17 isolates of genera Bacillus, Staphylococcus, Salmonella, Pseudomonas, Listeria and E. coli were evaluated for their biocide resistance profiles by determining their minimum inhibitory concentration (MIC) and also microbial biofilm eradicating concentration (MBEC) against commonly used biocides (iodophore, sodium hypochlorite, and benzalkonium chloride) in dairy/food industry. The Listeria, Salmonella and Staphylococcus exhibited highest MBEC against iodophore (550 ppm), Listeria against sodium hypochlorite (550 ppm); Bacillus, Pseudomonas, Listeria and consortia against benzalkonium chloride (650 ppm). Benzalkonium chloride and sodium hypochlorite were found most efficacious against all tested isolates and their consortia as they could reduce higher log counts after treatment in comparison to iodophore.

\section{Introduction}

Bacteria irreversibly attached to a substratum and embedded in an extracellular polymeric matrix that confers resistance for the involved microorganisms of their own synthesis forming a community of microorganisms is known as the biofilm (Donlan, 2002). Generally, biofilm maturation occurs in five distinguished phases: reversible adsorption, irreversible attachment, maturation I, maturation II and dispersion. Now, second phase of biofilm formation is irreversible attachment wherein bacteria adhere firmly to substrate surface and lose their mobility. Bacterial cells attach to each other and to the substrate surface and thus formation of bacterial micro colonies begins. This phase lasts for two hours (Maric and Wranes, 2007).

Microbial biofilms which form on all types of surfaces of technological systems in the dairy industry and on dairy farms adversely affect the quality and safety of final products, i.e. 
both foodstuffs and raw materials used for their production as besides bacteria insignificant to health, they may contain pathogenic microorganisms also. Thus, direct contact with raw materials or foodstuffs can cause secondary contamination due to which the product will become unsafe. Bacterial contamination that causes food decay and decreased quality is technologically important because of the fact that a number of microorganisms are alimentary pathogens, e.g. Staphylococcus aureus or Listeria monocytogenes and some others make a serious problem directly affecting human health when present in the food.

Outbreaks of pathogens associated with biofilms have been related to the presence of Listeria monocytogenes, Yersinia enterocolitica, Campylobacter jejuni, Salmonella spp. Staphylococcus spp. and Escherichia coli O157:H7 (Aarnela et al., 2007). Pathogenic microorganisms (from genera Bacillus, Streptococcus and Staphylococcus, Shigella, Escherichia, and Enterobacter aerogenes) participated in the biofilm formation on the surfaces of a postpasteurisation unit in a dairy plant. Several studies have reported the persistence of pathogenic bacteria on food and on medical equipments despite the use of cleaning and disinfection procedures (Brooks and Flint 2008; Simoes et al., 2010; Srinivasan et al., 2003). Poulsen (1999) reported that persistence of biofilms is probably due to the failure of disinfectant products, the inappropriate disinfection protocols and increased tolerance of bacteria due to adaptation to antimicrobial agents.

Biofilms are usually formed by various species of microorganism, which protect each other against the effects of biocidal (antibacterial) agents and are resistant to these agents. This is caused by the different resistance of respective microbial species against the agents used (Wirthlin et al., 2005).Sanitation regime, which meets the basic requirements, must be based on the knowledge of the particular conditions of the sanitised environment: (1) detected pathogens, colonising the cleaned surface;(2) knowledge of the aimed effects of the selected biocides against the pathogens;(3) chemical and physical properties of the surfaces;(4) hygienic design of technological systems (Salo et al., 2001). To control biofilm formation and its eradication, various strategies have been explored in dairy/food industry that includes of incorporation of biocides such as benzalkonium chloride (BKC), sodium hypochlorite (SH) and iodophore (ID) etc. in CIP system which is one of the most common strategies.

Most efficient strategy for actions against biofilm to ensure food safety and quality in the dairy food industries could be to clean and disinfect food contact surfaces before bacteria get attached firmly to these surfaces to form mature biofilms. Hence it follows that it is necessary to test first the effectiveness of the sanitation agents and sanitation procedures under model laboratory conditions, above all in the dairy industry and on farms. Therefore, present investigation was aimed to evaluate biofilm formation potential of pathogenic and spoilage bacteria of dairy niche as well as determination of their biocide resistance profile to emerge with better cleaning strategies to enhance food safety and quality and to control them.

\section{Materials and Methods}

\section{Bacterial cultures}

A total of 24 bacterial cultures including their standard strains were used in the present investigation. The 17 isolates belonging to six genera viz., Escherichia coli, Pseudomonas, Salmonella, Bacillus, Listeria and 
Staphylococcus were identified from biofilms of dairy niche points.

Determination of biofilm formation potential by biomass indicator staining method

Quantitative biofilm assay was performed by using crystal violet staining method using a 96 well microtitre plate. Briefly, all isolates were grown overnight in trypticase soy broth (TSB), inoculated at 1\% inoculum in $200 \mu 1$ of the same medium and plates were incubated at $37^{\circ} \mathrm{C} / 24 \mathrm{~h}$. Quantitative estimation of biofilm formation was determined by the method Stepanovic et al., (2007): non biofilm producer $=\mathrm{OD} \leq \mathrm{OD}_{\mathrm{C}}$; weak biofilm producer $=\mathrm{OD}_{\mathrm{C}}<\mathrm{OD} \leq 2 \times \mathrm{OD}_{\mathrm{C}}$; moderate biofilm producer $=2 \mathrm{xOD}_{\mathrm{C}}<\mathrm{OD} \leq 4 \mathrm{xOD}_{\mathrm{C}}$; strong biofilm producer $=4 \mathrm{xOD}_{\mathrm{C}}<\mathrm{OD}$. Subsequently on the basis of optical density, isolates were categorized as strong, moderate, weak and non-biofilm formers.

\section{In-vitro Biofilm Development on Stainless Steel chips}

The method of Milanov et al., (2009) was used with slight modifications for in-vitro biofilm development. In-vitro biofilm was developed on stainless steel (SS) chips of size $1 \times 1 \times 0.2 \mathrm{~cm}$. The $\mathrm{SS}$ chips for biofilm development were first treated by keeping in 1 $\mathrm{N} \mathrm{HNO}_{3}$ solution for 30 minutes to clean it properly and then autoclaved a $121^{\circ} \mathrm{C}$ at 15 PSI for $15 \mathrm{~min}$. The microbial cell concentrations of overnight grown cultures in TSB were adjusted to OD of $0.5 \mathrm{McFarland}$ standards which correspond to $10^{8} \mathrm{CFU} / \mathrm{ml}$. The individual SS chips were placed in each well of 6-well plate. The cell suspension of test strain was added @ 1\% into each well containing fresh $9 \mathrm{ml} \mathrm{TSB}$. These experiment was divided into two sets: set I (incubated at $37^{\circ} \mathrm{C} / 3 \mathrm{~h}$ ) and set II (incubated at $37^{\circ} \mathrm{C} / 48 \mathrm{~h}$ ). After incubation at $37^{\circ} \mathrm{C}$ for $3 \mathrm{~h}$ and $48 \mathrm{~h}$, chips were removed and washed with $3 \mathrm{ml}$ of phosphate buffer saline (PBS) to remove loosely attached cells and nutrients. The biofilm were scraped off from SS chips, by moving a sterile swab approximately 10 times over the biofilm area. The swab was then placed into a tube containing $5 \mathrm{ml}$ of physiological saline and vigorously vortexed for 2 minutes. Appropriate dilutions were prepared and plated out on nutrient agar for standard plate count.

Determination of minimum inhibitory concentration (MIC) and microbial biofilm eradicating concentration (MBEC)

The MIC and MBEC assay were carried out as per the following protocol. Respective stock solutions of benzalkonium chloride $\left(10^{6} \mathrm{ppm}\right)$, sodium hypochlorite $\left(10^{4} \mathrm{ppm}\right)$, and iodophore $\left(10^{4} \mathrm{ppm}\right)$ were appropriately diluted in tryptic soy broth (TSB) tubes to obtain required concentrations (0 to $400 \mathrm{ppm}$ in steps of 50 ppm for determination of minimum inhibitory concentration). Aliquots of $1 \mathrm{ml}$ TSB containing different biocides concentrations, were inoculated at $1 \%$ inoculum rate with overnight grown cultures and incubated at $37^{\circ} \mathrm{C} / 24 \mathrm{~h}$. Throughout the experiment, TSB tubes (without biocide) inoculated with respective cultures were used as positive controls, whereas un-inoculated TSB tubes (with biocides) as negative controls (Ac). After incubation, $200 \mu \mathrm{l}$ aliquots (in triplicate) of respective cultures were transferred to a fresh 96 microtitre well-plate and optical density at $620 \mathrm{~nm}$ was measured using microplate reader (Spectrostar-Nano). Inhibitory concentration end point was considered as lowest concentration of biocide at which absorbance of test (A) was less than or equal to that of un-inoculated control (Ac). The efficiencies of biocides (100-650 ppm in steps of $50 \mathrm{ppm}$ ) against biofilms of selected isolates were determined in terms of microbial biofilms eradication concentration (MBEC). 
To determine MBEC, contents of microtitre plate with 24 h old biofilm were decanted and treated with biocides for contact time of 30 min. Neutralizing solutions used were $0.5 \%$ sodium thiosulphate in phosphate buffer $(\mathrm{pH}$ 7.2) in case of sodium hypochlorite and iodophore, and tween lecithin (stock solution: 40 gm L- $\alpha$ lecithin and $0.5 \mathrm{ml}$ Tween-80 per litre working solution: $25 \mathrm{ml}$ of stock solution diluted $500 \mathrm{ml}$ in distilled water) in case of benzalkonium chloride. Neutralizing solutions were added to stop the activity of biocide after pre-determined contact of time. The plates were then decanted, rinsed gently with sterile water and stained using crystal violet. The bound dye was resolubilized in $33 \%$ glacial acetic acid, absorbance of which was read as described earlier. MBEC was considered as the concentration of biocide at which $\mathrm{A} \leq \mathrm{Ac}$.

\section{Efficacy evaluation of biocides on biofilm formation}

Biofilm were developed on SS chips as per Milanov et al., (2009) for individual selected strains of E. coli, Bacillus, Pseudomonas, Staphylococcus, Listeria, and Salmonella and their consortia for pre-decided incubation time $(0,0.5,1,1.5$ and $2 \mathrm{~h})$, which indicates of irreversible stage of biofilm formation. After incubation the chips were washed with $3 \mathrm{ml}$ of phosphate buffer saline (PBS) to remove loosely attached cells and nutrients. Biofilm forming bacteria were scraped off from SS chips by moving a sterile swab approximately 10 times over the biofilm area.

The swab was then placed into a tube with 5 $\mathrm{ml}$ of physiological saline and vigorously vortexed for 2 minutes. Appropriate dilutions were prepared and plated out on standard plate count on nutrient agar for viable bacterial counts to be considered as control counts without giving any biocide treatment at each specific incubation time. The treatment with biocides were performed as the whole surface of SS chips were covered with the specified biocides (iodophore, sodium hypochlorite and BKC) with MBEC confirmed concentration, (Table 2), for contact time of $30 \mathrm{~min}$ after each specific incubation times of irreversible stage as prescribed earlier. After treatment biocides were removed and their effect was inhibited using appropriate neutralizing solutions as mentioned earlier. Biofilm bacteria were scraped off from SS chips by moving a sterile swab approximately 10 times over the biofilm area.

The swab was then placed into a tube with 5 $\mathrm{ml}$ of maximum recovery diluent and vigorously vortexed for 2 minutes. Appropriate dilutions were made in sterile physiological saline, and the numbers of cells were determined by standard plate count on nutrient agar. The counts of control and counts after treatment were observed to check the biocides efficiency at different incubation time of irreversible stage for each target genera and their consortia biofilm.

\section{Statistical analysis}

The statistical analysis of data was carried out using SAS 9.3 (SAS Institute Inc., Cary, USA). Results were analysed and the significance level was calculated using Tukey's Studentized Range (HSD) test and results are considered significant at $(\mathrm{p}<0.05)$. The results were expressed as mean \pm standard deviation.

\section{Results and Discussion}

\section{Determination of biofilm formation potential by biomass indicator staining method}

A total of 24 cultures including six standard cultures and their consortia (comprising of one isolate of each genera) were checked for their biofilm formation potential. It was assayed by 
using 96 well polystyrene microtitre plate and indirect quantitation was carried out using crystal violet staining. The isolates and standard cultures were categorized into no (-), weak $(+)$, moderate $(++)$, and strong $(+++)$ biofilm formers as per the method of Stepanovic et al., (2004).

Amongst the tested strains, higher biofilm formation potential was observed for two Staphylococcus, two Pseudomonas and one Listeria isolates. Two of Salmonella isolates were designated as weak biofilm producers. Two isolates of E. coli and one of Bacillus showed moderate biofilm formation potentials.

\section{Biofilm formation potential on stainless steel chips}

Cell counts were determined after incubation period of 3 and $48 \mathrm{~h}$ at $37^{\circ} \mathrm{C}$. The observations on biofilm formation potential of isolates on SS chips are displayed in Table 1. The number of cells on stainless steel chips ranged from $10^{3}$ to $10^{6} \quad \log _{10} \mathrm{CFU} / \mathrm{cm}^{2}$ after $3 \mathrm{~h}$ of incubation and $10^{5}$ to $10^{8} \log _{10} \mathrm{CFU} / \mathrm{cm}^{2}$ after $48 \mathrm{~h}$ of incubation at $37^{\circ} \mathrm{C}$. The results are in corroboration with the research findings of Chae and Schraft (2001), who reported similar values for attachment of $L$. monocytogenes cells, on glass surface for $3 \mathrm{~h}$ incubation at $37^{\circ} \mathrm{C}$.

Among the entire target microflora, significantly higher biofilm formation potential, in terms of $\log _{10} \mathrm{CFU} / \mathrm{cm}^{2}$ was observed for Pseudomonas. Our findings in this regard are in accordance with the previous study by Klausen (2003), where Pseudomonas cultures exhibited strong biofilm formation owing to secondary attachment. Strain Staphylococcus (PC1 ST 4) had a lower attachment on SS chips after $3 \mathrm{~h}$ of incubation i.e. $3.23 \pm 0.132 \quad \log _{10} \quad \mathrm{CFU} / \mathrm{cm}^{2}$, which increased after $48 \mathrm{~h}$ incubation to $7.857 \pm 0.174$ $\log _{10} \mathrm{CFU} / \mathrm{cm}^{2}$ at $37^{\circ} \mathrm{C}$. In case of
Pseudomonas (PC2 Ps 3), the counts were $5.19 \pm 0.41 \log ^{10} \mathrm{CFU} / \mathrm{cm}^{2}$ after $3 \mathrm{~h}$ which to $7.965 \pm 0.174 \log ^{10} \mathrm{CFU} / \mathrm{cm}^{2}$ after $48 \mathrm{~h}$ incubation. These results are in accordance with the investigations of Chae and Schraft (2000), who experimentally proved that growth of L. monocytogenes in biofilm were independent of initial attachment. High biofilm formation potential on SS chips was observed for consortia after $3 \mathrm{~h}$ incubation as compared to individual strains, which showed almost two times higher log counts.

\section{Determination of inhibitory concentration of biocides against target strains}

The standard cultures along with one tested isolate from each chosen genera were selected for determination of their control on biofilm formation as per Table 2. The MIC and MBEC of dairy niche isolates against iodophore was observed in the range of 20-70 ppm and 250-550 ppm, respectively for target genera and their consortia. The MIC and MBEC values for sodium hypochlorite ranged from 100-200 ppm and 300-550 ppm, respectively for tested strains and their consortia. Our results regarding MBEC of sodium hypochlorite are in agreement with Souza et al., (2014) who stated that concentration of $250 \mathrm{mg} / \mathrm{L}$ of $\mathrm{SH}$ was not sufficient for complete removal of the cells of S. aureus from a biofilm on polypropylene and stainless steel surfaces.The MIC and MBEC values in case of benzalkonium chloride ranged from $150-250 \mathrm{ppm}$ and $500-650 \mathrm{ppm}$, respectively. Results on MIC, recorded that $E$. coli was highly susceptible and Pseudomonas most resistant against benzalkonium chloride.Our results are in agreement with Gilbert and Moore, (2005), who also noticed that some species of bacteria, notably Pseudomonas aeruginosa, are relatively insensitive to QAC biocides and it is thought to be related to a failure of compounds to penetrate the outer membrane. 
Table.1 Biofilm formation potential on SS chips of selected isolates of dairy niche

\begin{tabular}{|l|l|l|l|} 
S. No. & Genera & \multicolumn{2}{|l|}{$\log _{10} \mathrm{CFU} / \mathrm{cm}^{2}$ at $37^{\circ} \mathrm{C}($ mean value \pm SD) } \\
\cline { 3 - 4 } & after $3 \mathrm{~h}$ incubation & after 48 h incubation \\
\hline 1 & Bacillus & $3.23 \pm 0.28$ & $6.17 \pm 0.20$ \\
\hline 2 & Listeria & $3.34 \pm 0.075$ & $6.345 \pm 0.132$ \\
\hline 3 & Pseudomonas & $5.19 \pm 0.41$ & $7.857 \pm 0.23$ \\
\hline 4 & Staphylococcus & $3.23 \pm 0.132$ & $7.965 \pm 0.174$ \\
\hline 5 & E. Coli & $3.34 \pm 0.162$ & $6.85 \pm 0.29$ \\
\hline 6 & Salmonella & $3.73 \pm 0.25$ & $5.85 \pm 0.28$ \\
\hline 7 & Consortia & $6.65 \pm 0.34$ & $8.231 \pm 0.325$ \\
\hline
\end{tabular}

Table.2 MIC and MBEC of biofilm forming microbes of dairy niche

\begin{tabular}{|c|c|c|c|c|c|c|}
\hline Genera/isolate & \multicolumn{6}{|c|}{ MIC/MBEC of biocides (ppm) } \\
\hline \multicolumn{7}{|l|}{ 1. Bacillus } \\
\hline $\begin{array}{l}\text { Standard } \\
\text { Culture }\end{array}$ & \multicolumn{2}{|c|}{ Iodophore } & \multicolumn{2}{|c|}{$\begin{array}{l}\text { Sodium } \\
\text { hypochlorite }\end{array}$} & \multicolumn{2}{|c|}{$\begin{array}{l}\text { Benzalkonium } \\
\text { chloride }\end{array}$} \\
\hline & MIC & MBEC & MIC & MBEC & MIC & MBEC \\
\hline B. cereus ATCC 11778 & 20 & 350 & 200 & 400 & 200 & 650 \\
\hline ST B 1 & 30 & 300 & 250 & 450 & 250 & 650 \\
\hline \multicolumn{7}{|l|}{ 2. Pseudomonas } \\
\hline Ps. aeruginosa MTCC 741 & 20 & 300 & 200 & 450 & 250 & 650 \\
\hline PC2 Ps 3 & 30 & 300 & 250 & 450 & 250 & 600 \\
\hline \multicolumn{7}{|l|}{ 3. E. coli } \\
\hline E. coli ATCC 25922 & 20 & 250 & 100 & 300 & 150 & 500 \\
\hline PC2 EC 3 & 20 & 250 & 150 & 300 & 150 & 500 \\
\hline \multicolumn{7}{|l|}{ 4. Listeria Monocytogenes } \\
\hline $\begin{array}{l}\text { L. monocytogenes ATCC } \\
19115\end{array}$ & 70 & 550 & 150 & 550 & 200 & 650 \\
\hline PC2 Lis 3 & 70 & 550 & 150 & 550 & 200 & 600 \\
\hline \multicolumn{7}{|l|}{ 5. Salmonella } \\
\hline S. typhi NCDC 113 & 70 & 550 & 150 & 500 & 150 & 500 \\
\hline PC1 S 3 & 70 & 550 & 150 & 500 & 150 & 500 \\
\hline \multicolumn{7}{|l|}{ 6. Staphylococcus } \\
\hline S. aureus MTCC 3160 & 30 & 550 & 125 & 450 & 150 & 600 \\
\hline PC1 ST 4 & 40 & 600 & 150 & 450 & 200 & 650 \\
\hline \multicolumn{7}{|l|}{ 6. Consortia } \\
\hline Consortia & 20 & 350 & 150 & 350 & 250 & 650 \\
\hline
\end{tabular}

Consortia: Mixture of each selected genera isolate in same proportion, Sampling points: (PC1:Pre-chiller, PC2:Postchiller, ST: Storage tank valve, PO: Pasteurizer outlet), ppm: parts per million. 
Fig.1 Efficacy of biocides in controlling biofilms on stainless steel chips

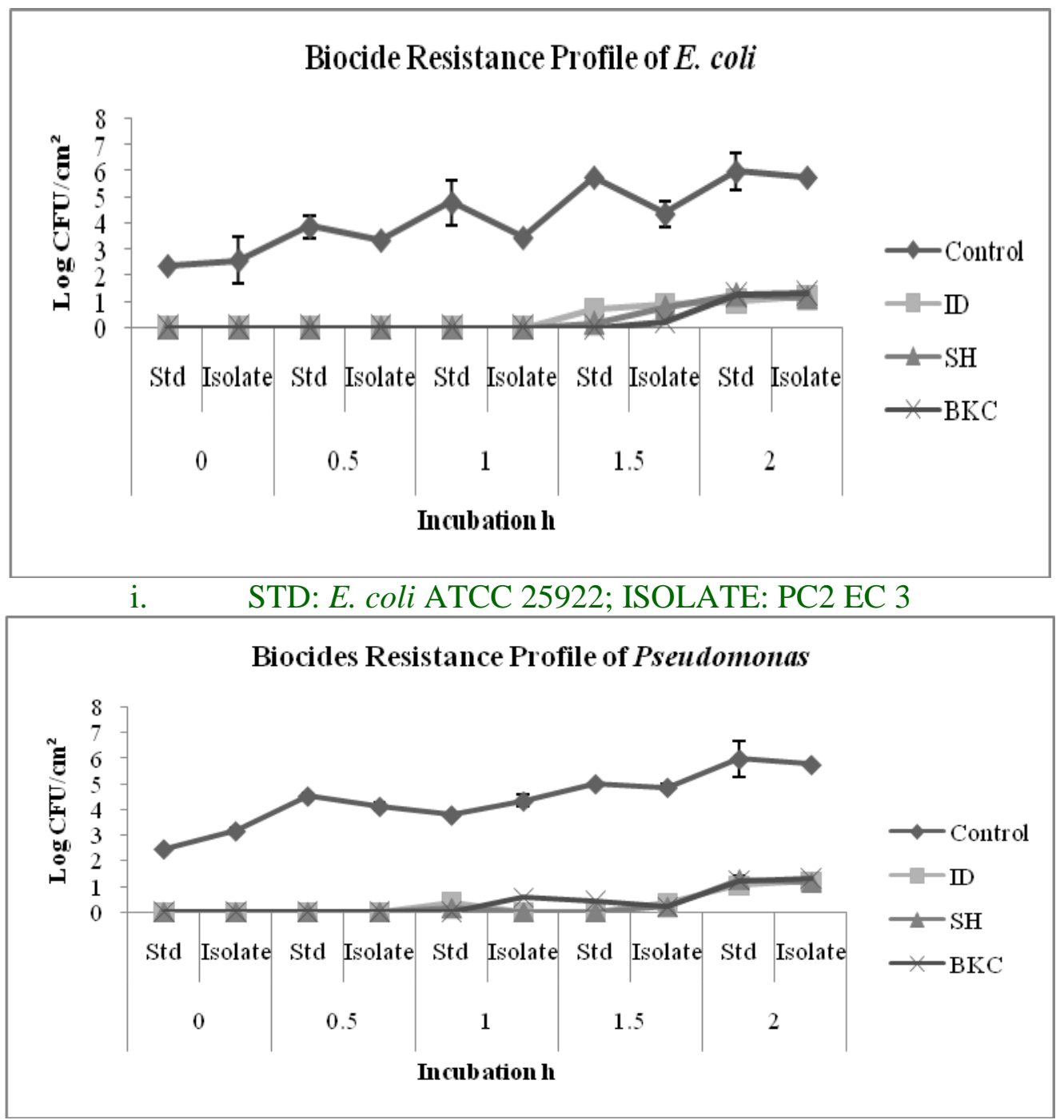

ii. STD: Ps. aeruginosa MTCC 741; ISOLATE: PC2 PS 3

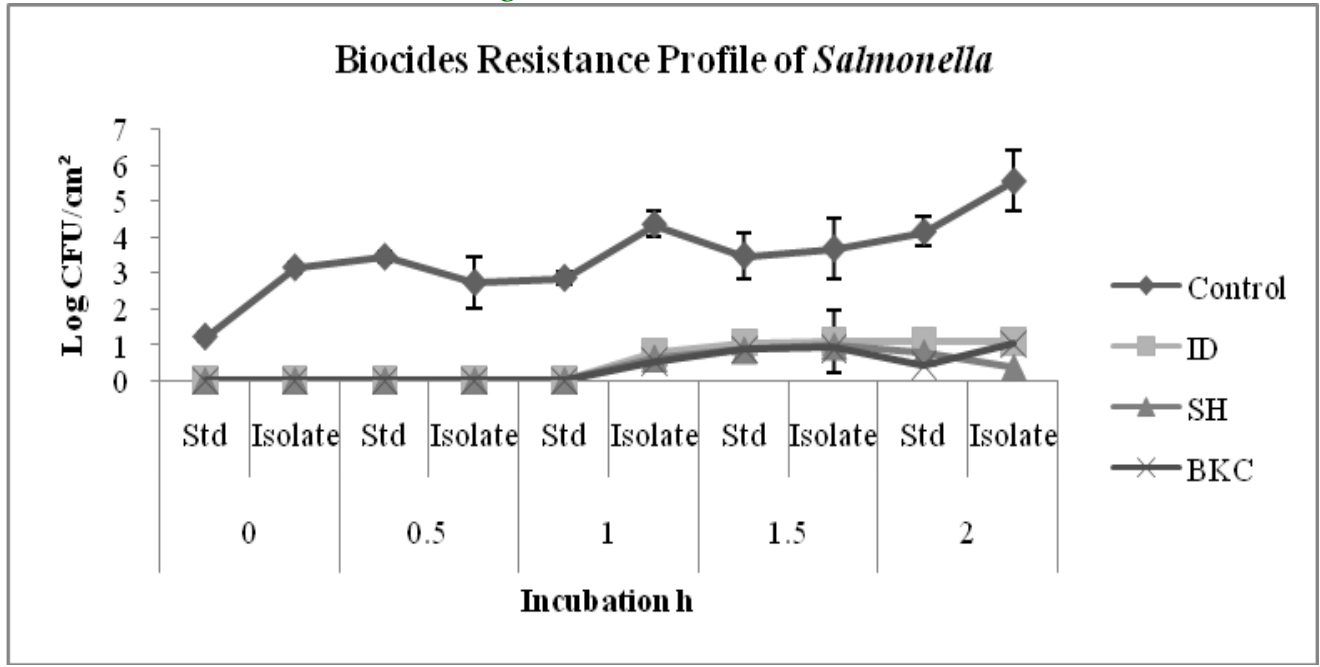

iii.

STD: S. typhi NCDC113; ISOLATE: PC1 S 3 


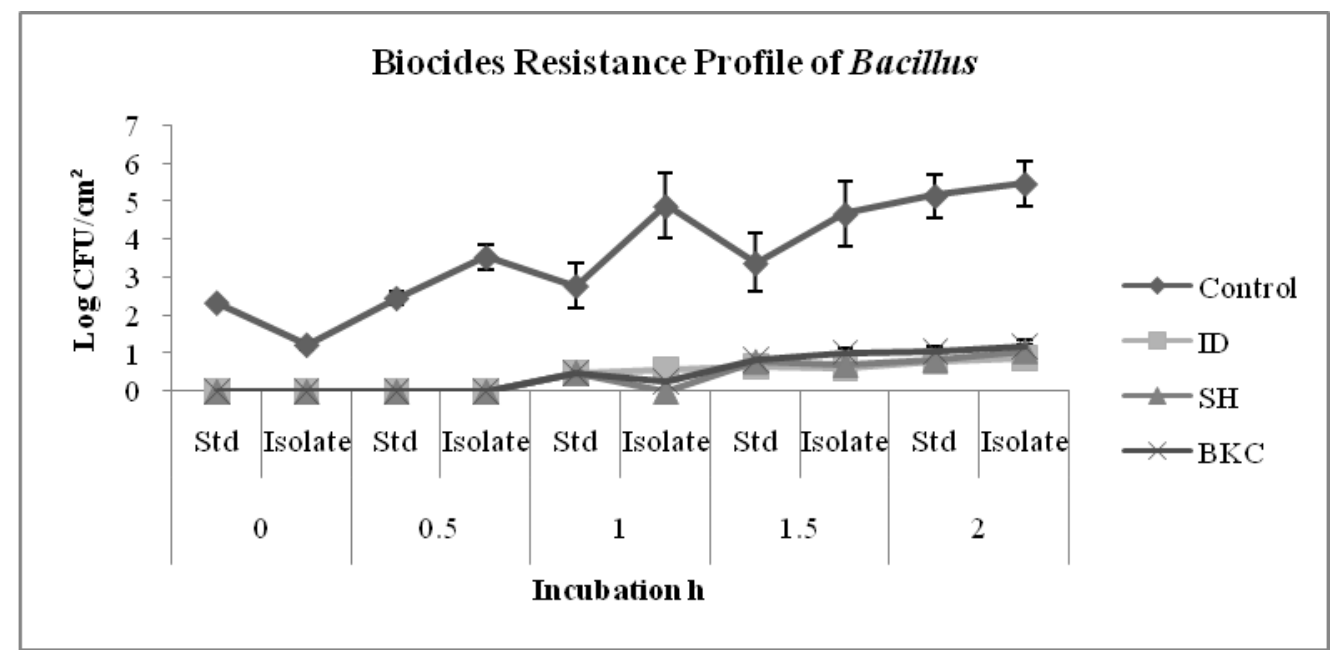

iv.

STD: $B$. cereus ATCC 11778; ISOLATE: ST B 1

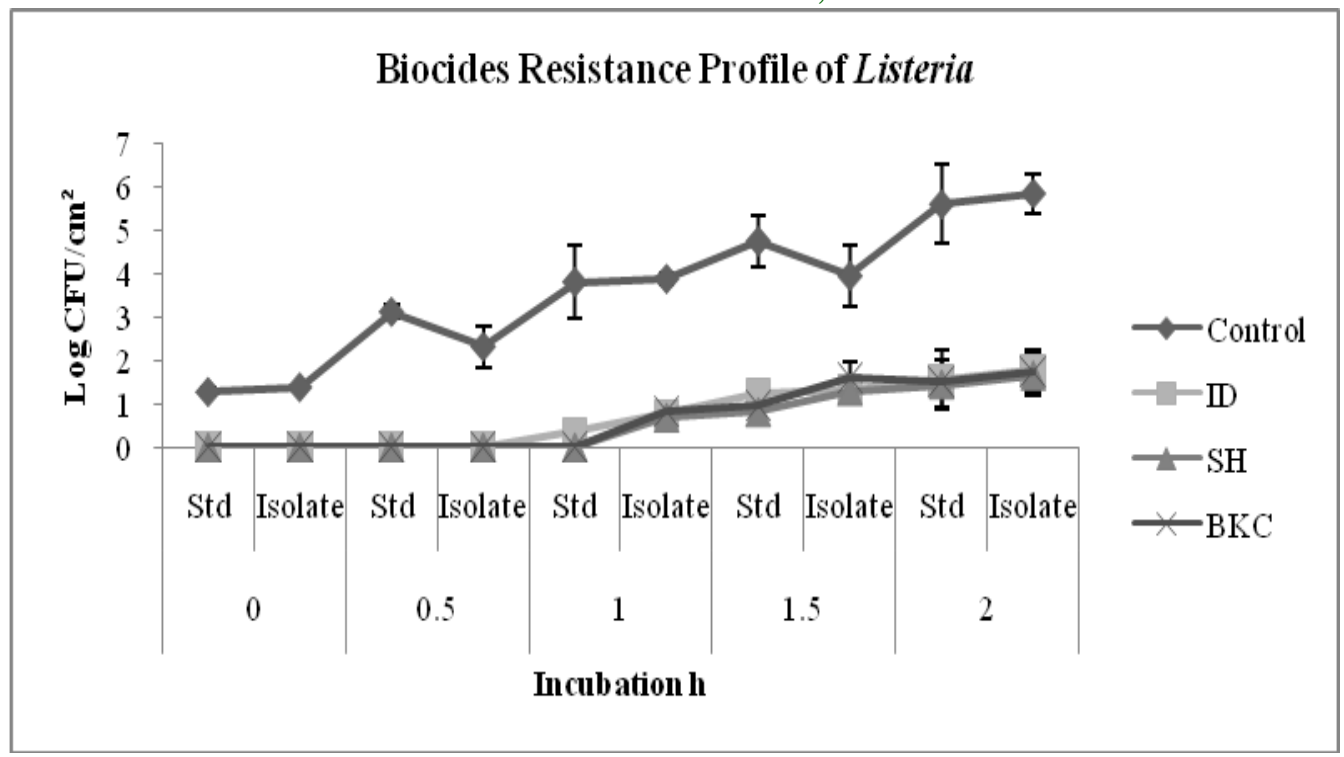

v. STD: L. monocytogenes ATCC19115; ISOLATE: PC2 LIS 3

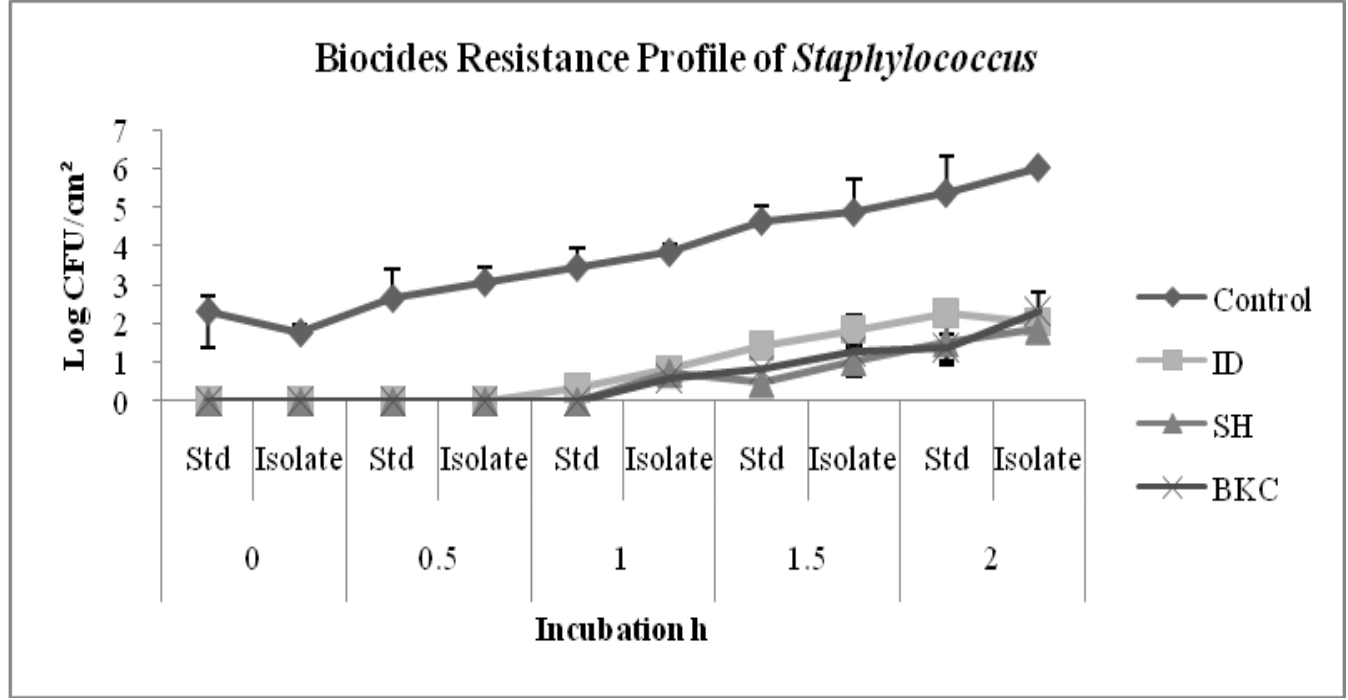

vi. STD: Staph. aureus MTCC 3160; ISOLATE:PC1 ST 4 


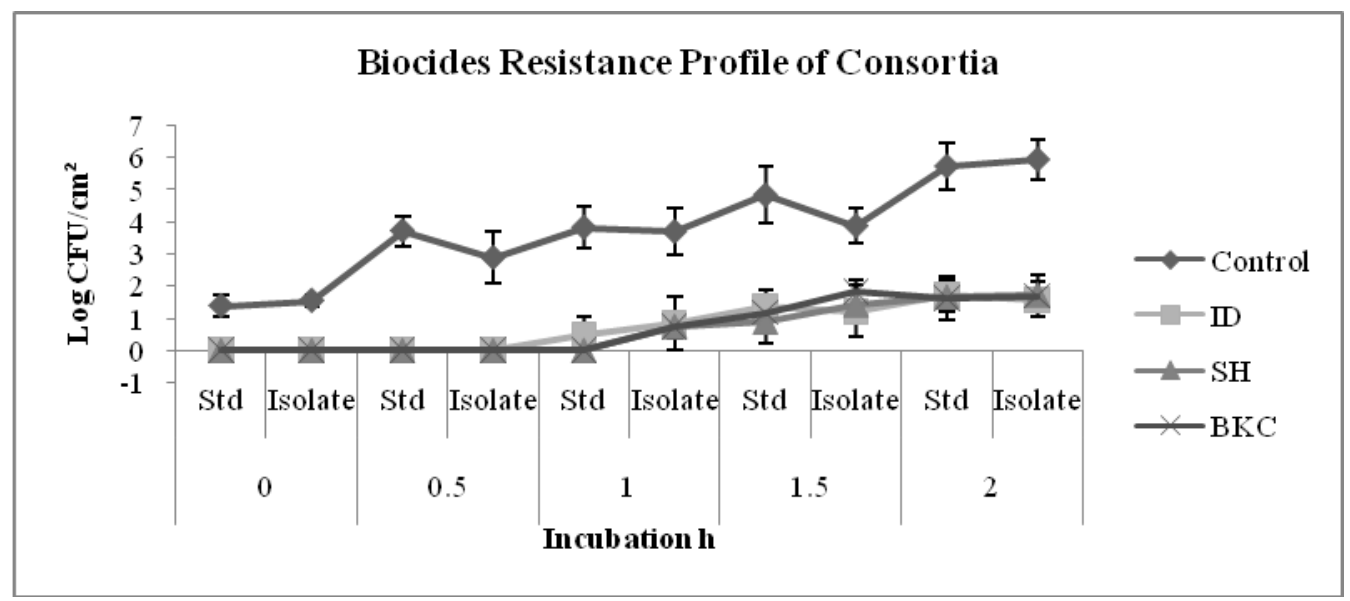

vii.

STD: Mixture of standard strains shown in above graphs; isolate: mixture of isolated strains shown in above graphs

ID: Iodophore (grey column), SH: Sodium Hypochlorite (dark grey column), BKC: Benzalkonium Chloride (black column), control (ash column). Effect of these disinfectants on biofilms of Std: standard culture and Isolate: selected strain grown on stainless steel chips of respective genera (shown as the graph head) and their consortia, at 0, 0.5, $1.0,1.5 \mathrm{~h}$. Data represent the mean of recovered viable and culturable cells count $\left(\log \mathrm{CFU} / \mathrm{cm}^{2}\right) \pm \mathrm{SD}$. Consortia: Mixture of each genera isolate in same proportion

\section{Efficacy evaluation of biocides on biofilm formation}

The efficacy of biocides to control biofilm formation was evaluated by incubating SS chips individually in TSB for pre-decided incubation time ( $0,0.5,1,1.5$ and $2 \mathrm{~h})$. These incubation times are representatives of irreversible stage in biofilm formation. The treatment with three biocides reduced 2-4 $\log _{10} \mathrm{CFU} / \mathrm{cm}^{2}$ counts of biofilm formed by isolate and their consortia at each incubation time. Bacillus cultures showed a significant inhibition when treated with ID and SH in controlling cell counts of the test organism as compared to BKC. The results of present study are in accordance with Ali and Maryam (2012), who reported that BKC was least effective against Bacillus cereus because of its ability to form spores. The spores of $B$. cereus have been reported to possess a pronounced ability to adhere to stainless steel, which draws attention in terms of food safety. Results on efficacy of biocide to control biofilms have been presented in Fig.1. These findings are in agreement with results of Pagedar et al., (2010), who also demonstrated that biofilms were most resistant to sodium hypochlorite and benzalkonium chloride and least to iodophore.

\section{Determination of biofilm formation potential by biomass indicator staining method}

The results obtained after analysis indicated that all tested isolates were able to form biofilm in microtitre plate. Our observations in this regard are in agreement with Klausen et al., (2003). The research findings for all the isolates are in agreement with the observations of Pagedar (2007), wherein she studied the development of multiple resistances and their transfer in potential food pathogens of food contact surface biofilms and stated strong biofilm (+++) formation by all selected isolates at $37^{\circ} \mathrm{C}$.

\section{Biofilm formation potential on stainless steel chips}

All the examined strains expressed the ability of attachment and growth on stainless steel surface when these were incubated in Tryptic 
Soy Broth (TSB) at $37^{\circ} \mathrm{C}$. The results on biofilm formation potential of isolates on stainless steel chips noted that all selected strains and their consortia possessed stability for biofilm formation on food contact surfaces. Data on biofilm formation of isolates from dairy niche after $48 \mathrm{~h}$ incubation, indicated that all strains showed increase of 2-4 $\log \mathrm{CFU} / \mathrm{cm}^{2}$ cell counts as compared to $3 \mathrm{~h}$ incubation indicative of final attachment on SS chips was independent of initial attachment. However, marginal difference was recorded in log counts of individual and consortia after $48 \mathrm{~h}$ incubation. These results are indicative of possible occurrence of associative growth among different strains.

\section{Determination of inhibitory concentration of biocides against target strains}

The results on MIC and MBEC demonstrated that E. coli was highly susceptible, whereas, Salmonella was highly resistant against iodophore as they showed highest MIC values, while for MBEC; isolates of Staphylococcus and Salmonella exhibited highest resistance against three biocides in the study and E. coli as least resistant. As per MIC, E. coli isolates were found highly susceptible, whereas, Bacillus and Pseudomonas were the most resistant against sodium hypochlorite. As far as MBEC is concerned, E. coli species were found to be highly resistant against sodium hypochlorite. The observations on MBEC for benzalkonium chloride demonstrated that, E. coli and Salmonella were highly susceptible whereas Bacillus, Pseudomonas, Listeria, and consortia, most resistant.

\section{Efficacy evaluation of biocides on biofilm formation}

Our in-vitro research findings on efficacy evaluation of biocides against biofilms on food contact surfaces (Stainless Steel chips) showed that sodium hypochlorite and benzalkonium chloride were more effective than iodophore to control preformed biofilms of 0.5 to $1.5 \mathrm{~h}$, however used concentrations of present study were higher than the recommended in-use concentrations. The results revealed that sodium hypochlorite and benzalkonium chloride were more effective in controlling biofilms formed by Gram's negative bacteria on SS chips of attachment time of $1.5 \mathrm{~h}$, as compared to iodophore which was least effective. These can be explained by the fact that penetration of QAC in biofilms was hindered probably due to the interactions of disinfectants with EPS of biofilm matrix which leads to an increased resistance of biofilm (Bridier et al., 2011; Davison et al., 2010). The research observations of the present investigations related to inhibition of biofilm of consortia revealed that sodium hypochlorite and benzalkonium chloride were found most effective as compared to iodophore in controlling biofilms on SS chips. Thus, Application of biocides at used concentrations remained ineffective against biofilms, which will not only compromise food quality and safety but also, would lead in emergence and spread of antimicrobial resistance.

It is possible to prevent the formation of bacterial populations resistant to the disinfection agents by the use of effective cleaning and disinfection procedures and thus prevent the development of hazardous environmental conditions in the food industry and on farms, in many cases. The observations on inhibition of biofilm formation which are at irreversible stage revealed that this can be inhibited by above mentioned biocides' treatment. Biocides used in this study resulted effective reduction in colony counts of minimum 2 log cycles and thus, these concentrations would be able to inhibit biofilm in their initial irreversible 
stage in dairy niche. Present study emphasizes the need of a systematic approach for selecting type and concentrations of biocides for application in dairy/food industry to control biofilm at their irreversible stage. The administration of above biocides at their MBEC level would also enable us to control contamination of dairy foods with spoilage and pathogenic microbes due to inhibition of biofilm. Thereby improving food safety and shelf-life of milk and milk products as well as minimise economic losses to dairy industry. Despite the fact, of successful attempts in vitro on biofilms, the in vivo mechanism is still poorly understood and requires the insights of the molecular mechanism of bacterial biofilms.

\section{Acknowledgement}

The authors acknowledge the financial support from Indian Council of Agricultural Research, New Delhi, India, in the form of network project "AMAAS". Authors also express their indebtness to Director, ICARNDRI, Karnal-132001, Haryana, India for providing infrastructure to carry out the research project.

\section{References}

Aarnela, K., Lunden, J., Korkeala, H., and Wirtanen, G. 2007. Susceptibility of Listeria monocytogenes strains to disinfectants and chlorinated alkaline cleaners at cold temperatures. Food Science and Technology. 40: 10411048

Ali, F., and Maryam, E. 2012. The disinfectant effects of Benzalkonium Chloride on some important foodborne pathogens. American-Eurasian J. Agric. \& Environ. Sci., 12(1): 23-29.

Bridier, A., Briandet, R., Thomas, V., and Dubois-Brissonnet, F.2011. Resistance of bacterial biofilms to disinfectants: a review. Biofouling. 27(9): 1017-1032.

Brooks, J. D., and Flint, S. H. 2008. Biofilms in the food industry: problems and potential solutions. Int $\mathrm{J}$ Food Sci Technol. 43(12): 2163-2176.

Chae, M. S., and Schraft, H. 2000. Comparative evaluation of adhesion and biofilm formation of different Listeria monocytogenes strains. International Journal of Food Microbiology. 62: 103111.

Chae, M. S., and Schraft, H. 2001. Cell viability of Listeria monocytogenes biofilms. Food Microbiol. 18:103-12.

Chmielewski, R. A. N., and Frank, J. F. 2006. Biofilm formation and control in food processing facilities. Comprehensive reviews on Food Science \& Food Safety. 2(1): 22-32.

Davison, W. M., Pitts, B., and Stewart, P. S. 2010. Spatial and temporal patterns of biocide action against Staphylococcus epidermidis biofilms. Antimicrob Agents Chemother. 54(7):2920-2927.

De Souza, E. L., Meira, Q. G. S., de Medeiros B., I., Athayde, A. J. A. A., da Conceicao M. L., and de Siqueira, J. J. P. 2014. Biofilm formation by Staphylococcus aureus from food contact surfaces in a meat-based broth and sensitivity to sanitizers. Brazilian Journal of Microbiology. 45(1):67-75.

Donlan, R. M. 2002. Biofilms: microbial life on surfaces. Emerg Infect Dis. 8(9): 881-890.

Gilbert, P. and Moore L. E. 2005. Cationic antiseptics: diversity of action under a common epithet. J Appl Microbiol. 99: 703-715.

Girard, L. P., Ceri, H., Gibb, A. P., Olson, M., and Sepandj, F. 2010. MIC versus MBEC to determine the antibiotic sensitivity of Staphylococcus aureus in peritoneal dialysis peritonitis. Peritoneal Dialysis International. 30(6): 652-656.

Klausen, M., Heydorn, A., Ragas, P., 
Lambertsen, L., Aaes-Jorgensen, A., Molin, S., and Tolker-Nielsen, T. 2003. Biofilm formation by Pseudomonas aeruginosa wild type, flagella and type IV pili mutants. Mol Microbiol. 48(6): 1511-24.

Maric, S. and Vranes, J. 2007.Characteristics and significance of microbial biofilm formation. Period Biol., 109(2):115121

Milanov, D., Asanin, R., Vidic, B., Katic, V., and Plavsa, N. 2009. Examination of the capabilities of attachment and biofilm formation of different Listeria monocytogenes strains. Biotechnology in Animal Husbandry. 25(5-6): 12551265.

Pagedar, A. 2007. Studies on the development of multiple resistances and their transfer in potential food pathogens within contact surface biofilms. Dissertation, National Dairy Research Institute, Karnal (Haryana), India.

Pagedar, A., Singh, J., and Batish, V. K.2010. Surface hydrophobicity, nutritional contents affect Staphylococcus aureus biofilms and temperature influences its survival in preformed biofilms. J Basic Microbiol. 50: S98-S106.

Poulsen, L. V. 1999. Microbial Biofilm in food processing. LWT-Food Sci Technol. 32(6): 321-326.

Salo, S., Mattila-Sandholm, T., and Wirtanen,
G. 2001. Do we need enhanced low pressure cleaning procedures for open process surfaces? In: Proceedings of $32^{\text {nd }}$ R3-Nordic Symposium on Contamination Control. Pp. 185-193.

Simoes, M., Simoes, L. C., and Vieira, M. J. 2010. A review of current and emergent biofilm control strategies. Food Sci Technol. 43(4): 573-583.

Srinivasan, A., Wolfenden, L. L., Song, X., Mackie, K., Hartsell, T. L., Jones, H. D., Diette, G. B., Orens, J. B., Yung, R. C., Ross, T. L., Merz, W., Scheel, P. J., Haponik, E. F., and Perl, T. M. 2003. An outbreak of Pseudomonas aeruginosa infections associated with flexible bronchoscopes. N Engl J Med. 348(3): 221-227.

Stepanovic, S., Vukovic, D., Hola, V., Di Bonaventura, G., Djukic, S., Cirkovic, I., and Ruzicka, F., 2007. Quantification of biofilm in microtiter plates: overview of testing conditions and practical recommendations for assessment of biofilm production by staphylococci. APMIS. 115(8): 891-9.

Wirthlin, M. R., Chen, P. K., and Hoover, C. I. 2005. A laboratory model biofilm fermenter: design and initial trial on a single species biofilm. Journal of Periodontology. 9: 1443-1449.

\section{How to cite this article:}

Mitali Makwana, Chand Ram Grover and Narendra Kumar. 2018. Biocides Resistance Profiles of Biofilm Forming Bacteria of Dairy Niche and their Control. Int.J.Curr.Microbiol.App.Sci. 7(02): 1194-1205. doi: https://doi.org/10.20546/ijcmas.2018.702.147 